\title{
ON THE TOPOLOGICAL STRUCTURE OF SIMPLY-CONNECTED ALGEBRAIC SURFACES
}

\author{
BY RICHARD MANDELBAUM AND BORIS MOISHEZON
}

Communicated by S. S. Chern, April 8, 1976

Suppose $X$ is a smooth simply-connected compact 4-manifold. Let $P=$ $C P^{2}$ and $Q=-C P^{2}$ be the complex projective plane with orientation opposite to the usual. We shall say that $X$ is completely decomposable if there exist integers $a, b$ such that $X$ is diffeomorphic to $a P \# b Q$.

By a result of Wall [W1] there always exists an integer $k$ such that $X$ \# $(k+1) P \# k Q$ is completely decomposable. If $X \# P$ is completely decomposable we shall say that $X$ is almost completely decomposable. In [MM1] we demonstrated that any nonsingular hypersurface of $C P^{3}$ is almost completely decomposable. In this paper we first announce generalizations of this result in two directions as follows.

THEOREM 1. Suppose $W$ is a simply-connected nonsingular complex projective 3-fold. Then there exists an integer $m_{0} \geqslant 1$ such that any hypersurface section $V_{m}$ of $W$ of degree $m \geqslant m_{0}$ which is nonsingular will be almost completely decomposable.

THEOREM 2. Let $V$ be a nonsingular complex algebraic surface which is a complete intersection. Then $V$ is almost completely decomposable.

IDEA OF PRoOF. The idea of the proofs is to degenerate $V$ (or $V_{m}$ ) to a pair of "less complicated" nonsingular surfaces crossing transversely and then use induction. The topological analysis of such a situation is then taken care of by Corollary 2.5 of [MM2] which states:

Corollary. Suppose $W$ is a compact complex manifold and $V, X_{1}, X_{2}$ are closed complex submanifolds with normal crossing in $W$. Let $S=X_{1} \cap X_{2}$ and $C=V \cap S$ and suppose as divisors $V$ is linearly equivalent to $X_{1}+X_{2}$. Let $\sigma: X_{2}^{\prime} \rightarrow X_{2}$ be the monoidal transformation of $X_{2}$ with center $C$. Let $S^{\prime}$ be the strict image of $S$ in $X_{2}^{\prime}$ and let $T_{2}^{\prime} \rightarrow S^{\prime}, T_{1} \rightarrow S$ be tubular neighborhoods of $S^{\prime}$ in $X_{2}^{\prime}$ and $S$ in $X_{1}$, respectively, with $H_{1}=\partial T_{1}$ and $H_{2}^{\prime}=\partial T_{2}^{\prime}$.

Then there exists a bundle isomorphism $\eta: H_{2}^{\prime} \longrightarrow H_{2}$ which reverses orientation on fibers such that $V$ is diffeomorphic to $\overline{X_{2}^{\prime}-T_{2}^{\prime}} \cup_{\eta} \overline{X_{1}-T_{1}}$.

Then if $V, X_{1}, X_{2}$ are simply connected 4-manifolds we can use [M] to

AMS (MOS) subject classifications (1970). Primary 57D55, 57A15, $14 \mathrm{~J} 99$. 
conclude that $V \approx X_{1} \# X_{2} \#(n-1) Q \# 2 g(P \# Q)$ where $n=\operatorname{card} C$ and $g=$ genus $S$. The proofs of Theorems 1 and 2 then conclude using an inductive argument.

This method gives us other results for which we establish some additional terminology. A field $F$ is called an algebraic function field of two variables over $\mathbf{C}$ if $F$ is a finitely-generated extension of $\mathbf{C}$ of transcendence degree two. Let $F$ denote the collection of all such fields. Then for $F \in F$ there exists a nonsingular algebraic surface whose field of meromorphic functions is $F$ (see $[\mathrm{Z}]$ ). We shall call any such nonsingular surface a model for $F$. It is then easy to see that given any two such models $V_{1}, V_{2}$ for $F$ their fundamental groups are isomorphic. Thus we define the fundamental group $\pi_{1}(F)$ for any $F \in F$ as the fundamental group of any model $V$ for $F$. We then let $F_{0}$ be the subcollection of simply-connected $F$ in $F$. For $F \in F_{0}$ we let $\mu(F)=\inf \{k \mid \exists$ a model $V$ for $F$ such that $V \# k P$ is completely decomposable\}. Using Wall's result previously mentioned, it can be seen that $\mu(F)$ is finite for any $F \in F_{0}$. If $F$ is a pure transcendental extension of $\mathbf{C}, \mu(F)=0$. If $\mu(F) \leqslant 1$ we shall call $F$ a topologically normal field. We now need

Definition 3. Let $L, K \in F$. Then $L$ is a satisfactory cyclic extension of $K$ if there exist models $V_{L}, V_{K}$ for $L$, resp. $K$ and a morphism $\Phi: V_{L} \rightarrow$ $V_{K}$ with discrete fibers whose ramification locus $R_{\Phi}$ is a nonsingular hypersurface section of $V_{K}$ whose degree is a multiple of $\operatorname{deg}(\Phi)$.

We then state

THEOREM 4. Let $K \in F_{0}$. Then there exists a satisfactory cyclic extension $L \in F_{0}$ of $K$ which is of degree 2 over $K$ and topologically normal.

In [M] it is further shown that if $K$ itself is topologically normal then so is any satisfactory cyclic extension. These two results motivate a partial order in $F_{0}$ defined as follows:

For $L, K \in F_{0}$ we shall say that $L$ is a satisfactorily resolvable extension of $K$ iff there exists a finite sequence of fields $L_{0}, \ldots, L_{n}$ in $F_{0}$ with $L_{0}=K$, $L_{i+1}$ a satisfactory cyclic extension of $L_{i}$ and $L_{n}=L$. We write $K<L$ if $L$ is a satisfactorily resolvable extension of $K$. Then $<$ induces a partial ordering on $F_{0}$. Our above results then say that in terms of this partial ordering every sufficiently "large" field $L$ is topologically normal.

Lastly we mention a purely topological counterpart of Theorem 4 .

THEOREM 5. Suppose $X$ is a smooth simply-connected 4-manifold. Let $F \in H_{2}(X, Z)$ with $F^{2} \neq 0$ and $F$ divisible by some integer $m \geqslant 2$. Then there exists a smooth compact simply connected 4-manifold $\widetilde{X}$ and a map $\Phi: \widetilde{X} \rightarrow$ $X$ exhibiting $\widetilde{X}$ as an $m$-fold branched cover over $X$ whose branch locus $R$ is a nonsingular representative of $F$ such that 
(1) If $F^{2}>0$ then $\widetilde{X} \# P$ is completely decomposable.

(2) If $F^{2}<0$ then $\widetilde{X} \# Q$ is completely decomposable.

\section{BIBLIOGRAPHY}

[M] R. Mandelbaum, Irrational connected sums and the topology of algebraic sur. faces (to appear).

[MM1] R. Mandelbaum and B. Moishezon, On the topological structure of nonsingular algebraic surfaces in $C P^{3}$, Topology 15 (1976), 23-40.

[MM2] - On the topology of simply-connected algebraic surfaces (to appear).

[W1] C. T. C. Wall, Diffeomorphisms of 4-manifolds, J. London Math. Soc. 39 (1964), 131-140. MR 29 \#626.

[W2] - On simply-connected 4-manifolds, J. London Math. Soc. 39 (1964), 141-149. MR 29 \#627.

[Z] O. Zariski, Algebraic surfaces, Ergebnisse Math. Grenzgebiete, Band 61, 2nd ed., Springer-Verlag, Berlin and New York, 1971.

DEPARTMENT OF MATHEMATICS, WEIZMANN INSTITUTE, REHOVOT, ISRAEL ISRAEL

DEPARTMENT OF MATHEMATICS, TEL AVIV UNIVERSITY, RAMAT-AVIV, 